\title{
EDITORIAL
}

\section{Finding suitable targets is the major obstacle to cancer gene therapy}

\section{Cancer Gene Therapy (2014) 21, 45-47; doi:10.1038/cgt.2014.3}

The durable complete cancer regressions observed in patients with metastatic melanoma receiving adoptive cell transfer (ACT) has demonstrated the power of this cell transfer approach for the treatment of cancer. ${ }^{1}$ The administration of autologous tumorinfiltrating lymphocytes (TILs) along with interleukin-2 following a lymphodepleting preparative regimen can lead to durable cancer regressions in $20-40 \%$ of patients with metastatic melanoma, most of whom were refractory to established regimens. ${ }^{2}$ Thus, attempts to develop lymphocytes with antitumor activity have become a major effort in studies of current cancer immunotherapy. The identification of naturally occurring cells with antitumor activity, for use in ACT, has thus far been limited to a small subgroup of cancers. Although TIL can be grown from virtually any cancer deposit, melanomas appear unique in the ability to reproducibly give rise to large numbers of cells with antitumor activity that can be readily detected by in-vitro assays. In-vitro sensitization of lymphocytes with tumor antigens can give rise to cells with antitumor activity, though successful examples of the use of these cells in the human are few. In an effort to generate antitumor lymphocytes for use in ACT in humans, attention has thus been turned to the genetic modification of normal circulating lymphocytes with retroviruses encoding either conventional alpha/beta $T$ cell receptors (TCRs) or chimeric antigen receptors (CARs) that can recognize cancers.

Although there have been early successes using this approach, the major obstacle confronting the widespread application of these studies has been the identification of suitable immunologic targets on cancer cells that can be attacked without injuring normal tissues. The seven major classes of antigens that have been targeted using cell transfer approaches are shown in Table 1.

The targeting of normal differentiation antigens that are overexpressed on cancers and minimally expressed on normal tissues have been attractive targets for cancer gene therapy. The success seen with the adoptive transfer of TILs in patients with metastatic melanoma was originally attributed to the common expression on these TILs of receptors that recognize melanoma/ melanocyte differentiation antigens such as MART- $1^{3}$ and gp-100, ${ }^{4}$ though this now does not appear to be correct. Toxicities to

Table 1. Categories of antigens targeted by adoptive cell therapy

1. Differentiation antigens overexpressed on cancers compared with normal tissue (MART-1, CEA, Her-2)

2. Antigens expressed on cancers and on non-essential normal tissues (CD19, thyroglobulin)

3. Shared non-mutated antigens unique to cancer (cancer-testes antigens)

4. Shared mutations unique to each cancer type (EGFRvIII)

5. Viral antigens on cancers caused by viruses (HPV E6 and E7, LMP1/2)

6. Critical components of the tumor stroma (VEGFR-2, FAP)

7. Driver or random mutations unique to each individual cancer normal melanocyte-bearing tissues in patients treated with TILs were rarely seen despite the induction of major cancer regressions. ${ }^{2}$ However, targeting of metastatic melanoma using $T$ cells genetically engineered with high-affinity receptors targeting the MART-1 and gp-100 antigens led to severe toxicities in the eye and ear, normal tissues that express melanocytes, thus suggesting that TIL were mediating antitumor effects by targeting different antigens. ${ }^{5,6}$ Although the resulting uveitis and hearing loss that resulted from this treatment could be ameliorated by the local administration of steroids, these toxicities have largely led to the abandonment of targeting these melanoma/melanocyte antigens for the treatment of patients with metastatic melanoma. As will be discussed later, recent studies have shown that the antitumor activity of TILs in patients with melanoma is due to the ability of these cells to target mutated antigens expressed on each individual cancer. ${ }^{7}$

Attempts to target gastrointestinal cancers overexpressing another differentiation protein, carcinoembryonic antigen (CEA), were severely limited by the colitis that occurred when highaffinity cells bearing receptors against CEA were administered. ${ }^{8}$ Although ongoing studies are using CARs to target other differentiation antigens such as mesothelin and Her-2 that are presented on the cell surface, the exquisite sensitivity of highaffinity $T$ cells is also likely to lead to normal tissue toxicities that will limit the application of cells targeting these types of differentiation antigens. ${ }^{9}$

Since cancer and normal tissues can share normal non-mutated differentiation antigens, a related approach is the targeting of normal antigens on cancers that are shared by non-essential normal tissues whose destruction would represent a tolerable price to pay for the elimination of metastatic cancer deposits. The predominant example of this category of targets is the treatment of patients with lymphomas and leukemias by targeting the CD19 molecule also expressed on normal B cells. A highly successful approach to the treatment of these advanced leukemias and lymphomas by targeting CD19 was first reported in 2010 (refs. 10,11) and was accompanied by the elimination of normal B cells, a toxicity that can largely be overcome by the administration of immunoglobulin preparations. The development of TCRs against thyroglobulin expressed on some thyroid cancers as well as normal thyroid tissue is being explored since hormonal replacement can substitute for destruction of the thyroid. Attempts are underway to identify tissue specific antigens expressed on additional cancers as well as normal tissues that are non-essential for life, such as the prostate, the ovary and the breast. Destruction of the normal epithelial tissues of these organs would represent a reasonable toxicity if successful cancer treatment could be achieved.

Cancer-testes antigens represent a group of proteins that are expressed during fetal development, are often epigenetically silenced in normal tissues, but are re-expressed in $20-80 \%$ of common epithelial cancers such as those of the bladder, lung, ovary, liver and others. Although some cancer-testes antigens such as NY-ESO-1 appear to be uniquely expressed only in cancers, many cancer-testes antigens do have some limited expression in normal tissues. The successful targeting of NY-ESO-1 by autologous lymphocytes genetically engineered to express high-affinity TCRs 
against this antigen has led to dramatic regressions in patients with metastatic synovial cell sarcoma and metastatic melanoma. ${ }^{12}$ Other solid tumors that express NY-ESO-1 are currently being studied as susceptible targets for this cell transfer therapy.

There are over 100 cancer-testes antigens and their low-level expression on normal tissues can represent a source of significant toxicity if this is not recognized. Thus, although MAGE-A3 is not expressed on normal tissues, an HLA-A2 restricted epitope on MAGE-A3 was similar to one on MAGE-A12, and thus targeting this epitope unexpectedly led to severe neurologic toxicity due to the expression of MAGE-A12 in the brain. ${ }^{13}$ Thus, before attacking any putative cancer antigen, a careful analysis of the entire human genome is essential to test whether there is expression or crossreactivity of the targeted sequences on normal tissues.

A different problem arose in targeting an HLA-A1 restricted epitope of MAGE-A3 when amino acids in the variable CDR combining regions of the TCR were substituted to increase the affinity of the receptor. ${ }^{14}$ Thus, although MAGE-A3 was not expressed in normal tissues, the modification of the TCR introduced new reactivities against a normal protein found in heart muscle that led to the death of two patients. Modified TCRs are not subjected to negative selection in the thymus and thus, reactivity to normal tissues can be introduced.

An ideal source of antigens to target using genetically modified lymphocytes are shared mutations that are unique to each cancer type and are not found on normal tissues. Common mutations such B-RAF in melanoma or K-RAS in pancreatic and other cancers would represent ideal targets for cell transfer immunotherapy, although, thus far, TCR or CAR recognizing these mutations have not been identified. A prominent example of a mutation shared among a single cancer type is the Epidermal Growth Factor Receptor variant 3 (EGFRvIll) mutation that is expressed in approximately $40 \%$ of high-grade glioblastomas. ${ }^{15}$ This mutation in the EFGR on the surface of glioblastomas results from an inframe deletion of exons 2-7, which results in a truncated extracellular ligand binding domain. This mutation results in constitutive activation of EGFR and leads to increased proliferation, invasion and motility of these mutated cells. Monoclonal antibodies against EGFRvIll have been developed and used to devise a CAR attacking this cell surface component. Dose escalation trials using this CAR in patients with glioblastomas are in progress in the Surgery Branch, National Cancer Institute. Similarly, viruses involved in oncogenesis often result in the expression of foreign viral proteins on cancers and not normal tissues. Antigens from the human papillomavirus (HPV) molecules E6 and E7 present new cell surface epitopes and TCRs are being developed to attack these components. Similarly, Epstein-Barr virus latent membrane protein-1 (LMP1) and LMP2 can present attractive targets for immunotherapy because of the lack of expression of these viral antigens on normal cells. ${ }^{16}$

The cancer cell represents only one part of the tumor mass and there are critical components of the tumor stroma that may also be susceptible to attack. Molecules such as the Vascular Endothelial Growth Factor Receptor-2 (VEGFR-2) which is overexpressed on tumor vasculature or Fibroblast Activation Protein (FAP) which is overexpressed on invading activated fibroblasts within the tumor stroma can be targeted though these normal non-mutated molecules have low expression on normal tissues, ${ }^{17,18}$ which may result in limiting toxicities.

All cancers contain multiple unique mutations and future progress in cancer gene therapy will likely result from the immunologic targeting of these mutated proteins. ${ }^{19}$ Melanomas contain an unusually large number of mutations probably due to the mutagenic action of ultraviolet light on the skin. The unique ability of TILs from melanoma to cause durable cancer regressions appears to result from attack against these individual cancer mutations. Smoking-induced lung cancers and cancers in patients with mutations in mismatch repair genes also have large numbers of sporadic mutations. The successful attack against unique antigens on melanoma suggests that TILs or gene-modified lymphocytes recognizing unique mutations on other cancers might be effective for use in therapy as well. Most other solid cancers, however, have anywhere from one-fifth to one-tenth the number of mutations compared with those present in melanoma and the targeting of these mutations represents a daunting problem. T cells reacting with sporadic or driver mutations on most cancers are likely to be infrequent though techniques for the identification of unique exomic mutations on individual cancers and the development of methods for obtaining $T$ cells against them are rapidly being developed. ${ }^{12}$

The ability to genetically modify lymphocytes has opened a new area of cancer therapy. The introduction of genes encoding conventional TCRs, as well as chimeric receptors represents only one aspect of this approach. It is also possible to genetically alter $T$ cells by introducing additional genes that affect cell function rather than recognition. Thus, in experimental models, the introduction of a gene encoding a single-chain interleukin-12 can significantly improve the anti-cancer effects of adoptively transferred T cells. ${ }^{20}$ Other approaches such as the introduction of genes encoding anti-apoptotic molecules such as Bcl-2, trafficking molecules such as CD62L or molecules that improve cell survival such as telomerase represent additional aspects of cancer gene therapy that will likely increase the anti-cancer impact of cell transfer approaches in the future.

Steven A Rosenberg E-mail: sar@nih.gov

\section{REFERENCES}

1 Rosenberg SA. Cell transfer immunotherapy for metastatic solid cancer-what clinicians need to know. Nat Rev Clin Oncol 2011; 8: 577-585.

2 Rosenberg SA, Yang JC, Sherry RM, Kammula US, Hughes MS, Phan GQ et al. Durable complete responses in heavily pretreated patients with metastatic melanoma using T-cell transfer immunotherapy. Clin Cancer Res 2011; 17: 4550-4557.

3 Kawakami Y, Eliyahu S, Delgado CH, Robbins PF, Rivoltini L, Topalian SL et al. Cloning of the gene coding for a shared human melanoma antigen recognized by autologous T cells infiltrating into tumor. Proc Natl Acad Sci USA 1994; 91: 3515-3519.

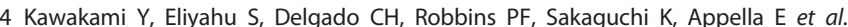
Identification of a human melanoma antigen recognized by tumor infiltrating lymphocytes associated with in vivo tumor rejection. Proc Natl Acad Sci USA 1994; 91: 6458-6462.

5 Morgan RA, Dudley ME, Wunderlich JR, Hughes MS, Yang JC, Sherry RM et al. Cancer regression in patients after transfer of genetically engineered lymphocytes. Science 2006; 314: 126-129.

6 Johnson LA, Morgan RA, Dudley ME, Cassard L, Yang JC, Hughes MS et al. Gene therapy with human and mouse T-cell receptors mediates cancer regression and targets normal tissues expressing cognate antigen. Blood 2009; 114: 535-546.

7 Robbins PF, Lu YC, El-Gamil M, Li YF, Gross C, Gartner J et al. Mining exomic sequencing data to identify mutated antigens recognized by adoptively transferred tumor-reactive T cells. Nat Med 2013; 19: 747-752.

8 Parkhurst MR, Yang JC, Langan RC, Dudley ME, Nathan DA, Feldman SA et al. $T$ cells targeting carcinoembryonic antigen can mediate regression of metastatic colorectal cancer but induce severe transient colitis. Mol Ther 2011; 19: 620-626.

9 Morgan RA, Yang JC, Kitano M, Dudley ME, Laurencot CM, Rosenberg SA. Case report of a serious adverse event following the administration of T cells transduced with a chimeric antigen receptor recognizing ERBB2. Mol Ther 2010; 18: 843-851.

10 Kochenderfer JN, Wilson WH, Janik JE, Dudley ME, Stetler-Stevenson M, Feldman $\mathrm{SA}$ et al. Eradication of B-lineage cells and regression of lymphoma in a patient treated with autologous T cells genetically engineered to recognize CD19. Blood 2010; 116: 4099-4102.

11 Kochenderfer JN, Rosenberg SA. Treating B-cell cancer with T cells expressing anti-CD19 chimeric antigen receptors. Nat Rev Clin Oncol 2013; 10: 267-276.

12 Robbins PF, Morgan RA, Feldman SA, Yang JC, Sherry RM, Dudley ME et al. Tumor regression in patients with metastatic synovial sarcoma and melanoma using genetically engineered lymphocytes reactive with NY-ESO-1. J Clin Oncol 2011; 29: 917-924.

13 Morgan RA, Chinnasamy N, Abate-Daga D, Gros A, Robbins PF, Zheng Z et al. Cancer regression and neurological toxicity following anti-MAGE-A3 TCR gene therapy. J Immunother 2013; 36: 133-151. 
14 Cameron BJ, Gerry AB, Dukes J, Harper JV, Kannan V, Bianchi FC et al. Identification of a Titin-derived HLA-A1-presented peptide as a cross-reactive target for engineered MAGE A3-directed T cells. Sci Transl Med 2013; 5: 197 ra103.

15 Morgan RA, Johnson LA, Davis JL, Zheng Z, Woolard KD, Reap EA et al. Recognition of glioma stem cells by genetically modified T cells targeting EGFRvll and development of adoptive cell therapy for glioma. Hum Gene Ther 2012; 23: 1043-1053.

16 Bollard CM, Gottschalk S, Torrano V, Diouf O, Ku S, Hazrat Y et al. Sustained complete responses in patients with lymphoma receiving autologous cytotoxic T lymphocytes targeting Epstein-Barr virus latent membrane proteins. J Clin Oncol 2013 (e-pub ahead of print 16 December 2013).
17 Chinnasamy D, Yu Z, Theoret MR, Zhao Y, Shrimali RK, Morgan RA et al. Gene therapy using genetically modified lymphocytes targeting VEGFR-2 inhibits the growth of vascularized syngenic tumors in mice. J Clin Invest 2010; 120: 3953-3968.

18 Tran E, Chinnasamy D, Yu Z, Morgan RA, Lee CC, Restifo NP et al. Immune targeting of fibroblast activation protein triggers recognition of multipotent bone marrow stromal cells and cachexia. J Exp Med 2013; 210: 1125-1135.

19 Vogelstein B, Papadopoulos N, Velculescu VE, Zhou S, Diaz Jr LA, Kinzler KW. Cancer genome landscapes. Science 2013; 339: 1546-1558.

20 Kerkar SP, Muranski P, Kaiser A, Boni A, Sanchez-Perez L, Yu Z et al. Tumor-specific CD8 + T cells expressing interleukin-12 eradicate established cancers in lymphodepleted hosts. Cancer Res 2010; 70: 6725-6734. 